\title{
Truncation effects in the functional renormalization group study of spontaneous symmetry breaking
}

\author{
N. Defenu, ${ }^{a, b}$ P. Mati, ${ }^{c, d, e}$ I.G. Márián, ${ }^{d}$ I. Nándori ${ }^{d, f, g}$ and A. Trombettoni ${ }^{b, a, h}$ \\ ${ }^{a} S I S S A$, \\ Via Bonomea 265, Trieste, I-34136 Italy \\ ${ }^{b}$ CNR-IOM DEMOCRITOS Simulation Center, \\ Via Bonomea 265, Trieste, I-34136 Italy \\ ${ }^{c}$ Budapest University of Technology and Economics, Department of Theoretical Physics, \\ Budapest, H-1111 Hungary \\ ${ }^{d}$ University of Debrecen, \\ P.O. Box 105, Debrecen, H-4010 Hungary \\ ${ }^{e}$ Eötvös University, Department of Atomic Physics, \\ Budapest, $\mathrm{H}$-1117 Hungary \\ ${ }^{f}$ MTA-DE Particle Physics Research Group, \\ P.O. Box 51, Debrecen, H-4001 Hungary \\ ${ }^{g}$ MTA Atomki, \\ P.O. Box 51, Debrecen, H-4001 Hungary \\ ${ }^{h}$ INFN, Sezione di Trieste, \\ Via Bonomea 265, Trieste, I-34136 Italy \\ E-mail: ndefenu@sissa.it, matipeti@gmail.com, \\ marianistvangabor@gmail.com, nandori@atomki.mta.hu, andreatr@sissa.it
}

ABSTRACT: We study the occurrence of spontaneous symmetry breaking (SSB) for $O(N)$ models using functional renormalization group techniques. We show that even the local potential approximation (LPA) when treated exactly is sufficient to give qualitatively correct results for systems with continuous symmetry, in agreement with the Mermin-Wagner theorem and its extension to systems with fractional dimensions. For general $N$ (including the Ising model $N=1$ ) we study the solutions of the LPA equations for various truncations around the zero field using a finite number of terms (and different regulators), showing that SSB always occurs even where it should not. The SSB is signalled by Wilson-Fisher fixed points which for any truncation are shown to stay on the line defined by vanishing mass beta functions.

Keywords: Field Theories in Lower Dimensions, Spontaneous Symmetry Breaking, Renormalization Group, Renormalization Regularization and Renormalons

ARXiv EPrint: 1410.7024 


\section{Contents}

1 Introduction 1

2 Functional renormalization group for the $O(N)$ model 3

3 The truncated $O(N)$ model $\left(N<\infty, N_{\mathrm{CUT}}<\infty\right)$

4 The spherical model without truncations $\left(N=\infty, N_{\mathrm{CUT}}=\infty\right) \quad 9$

5 The $O(N)$ model without truncations $\left(N<\infty, N_{\mathrm{CUT}}=\infty\right) \quad 11$

$5.1 d>2 \quad 13$

$5.2 d<2 \quad 13$

$5.3 d=2 \quad 14$

6 Conclusions 14

$\begin{array}{ll}\text { A Regulator functions } & 15\end{array}$

B Spontaneous symmetry breaking at finite $N \quad 16$

\section{Introduction}

Spontaneous symmetry breaking (SSB) is a cornerstone concept in a variety of systems, from field theory and particle physics to statistical mechanics and interacting lattice models. The study of the occurrence of SSB plays a crucial role in the theory of phase transitions and in the characterization of ordered phases and it highlights the interplay between SSB and the dimensionality of the system: this interplay is customarily expressed by defining the lower critical dimension $d_{\mathrm{L}}$ as the dimension for which SSB cannot occur at finite temperature [1].

A celebrated exact result connecting SSB and dimensionality is provided by the Mermin-Wagner theorem [2-4]. According to the Mermin-Wagner theorem a continuous symmetry cannot be spontaneously broken in two dimensions: $d_{\mathrm{L}}=2$. This theorem has been formulated for classical systems [2] and then extended to quantum systems [3, 4]. For magnetic systems with continuous symmetry it rules out the possibility of having a nonvanishing magnetization at finite temperature in two dimensions, and for $2 d$ interacting Bose gases predicts that no Bose-Einstein condensation occurs at finite temperature [3] (for Bose gases this result has been extended to zero temperature [5]). As well known, even though the Mermin-Wagner theorem rules out SSB and the existence of a local order parameter in two dimensions, nonetheless the Berezinskii-Kosterlitz-Thouless transition may yet occur for the U(1) symmetry and it signaled by the algebraic behaviour of correlation functions in the low temperature phase [6].

The Mermin-Wagner theorem for the $O(N)$-symmetric scalar field theory states that for $N \geq 2$ in two dimensions no SSB occurs. Although originally formulated in integer dimensions, this result was later extended to graphs with fractional dimension $[7,8]$ : in 
this way one can explicitly show that for $N \geq 2$ there is no SSB for $d \leq 2$, with $d$ being real, while SSB occurs for $d>2$ [9]. In [10] the study of how $O(N)$ universality classes depends continuously on the dimension $d$ (and as well on $N$ ), in particular for $2<d<3$, was recently presented. The Ising model, i.e. the $N=1$ case, is different from $O(N)$ models with continuous symmetry $(N \geq 2)$ because the symmetry is discrete: in two dimensions it notoriously has a finite temperature phase transition [11] and it can be shown that this happens for $d \geq 2$ with $d$ real [12]. The large $N$-limit of $O(N)$ models is also interesting because for $N \rightarrow \infty$ it is equivalent to the spherical model [13], which is exactly solvable [14].

The $O(N)$ model represents then an ideal playground to study the interplay of SSB and dimensionality and to test whether (and how) the appearance of SSB depends on the approximation schemes. A powerful method used to consider the phase structure of a model, and consequently to study the appearance of SSB, is the functional renormalization group (FRG) method [15-23]. The $O(N)$ model has extensively studied using FRG approaches: as relevant for our purposes, we mention it was used to study as a function of dimension critical exponents of $O(N)$ models $[10,24,25]$ and to investigate truncation effects and the regulator-dependence of the FRG equation [26-37], while a FRG study of the critical exponents of the Ising model for $d<2$ was presented in [24]. The study of single-particle quantum mechanics can be seen as a "low-dimensional" statistical mechanics model: FRG studies addressed double well potential and quantum tunneling [38, 39] and quartic anharmonic oscillators [40].

In the FRG framework one has to solve an integro-differential equation valid for functionals which is usually handled resorting to approximations. It is in general of great importance to know "how good" are the used approximations and to test them against exact results. An approximation commonly used is the Local Potential Approximation (LPA), in which the wave-function renormalization and higher derivative terms in the effective action are discarded, resulting in a vanishing anomalous dimension [16-23]. Furthermore, one often treats LPA introducing further approximations via the introduction of a finite number of couplings, defined as the Taylor coefficient of an expansion of the potential around the zero (or the minimum) of the field, and studying their renormalization group (RG) flow.

In this paper our goal is two-fold: i) from one side we aim at discussing what level of approximation is needed to reproduce the Mermin-Wagner theorem and to show that no SSB occurs for $d \leq 2$ with $d$ real and $N \geq 2$, ii) from the other we intend to investigate how truncation affects the occurrence or non-occurrence of SSB comparing/contrasting the obtained findings with the exact prediction of the Mermin-Wagner theorem. Our findings for systems with continuous symmetry can be summarized as follows:

i) LPA, when treated exactly, is enough to reproduce the Mermin-Wagner theorem;

ii) defining the couplings as the coefficients of a Taylor expansion of the effective potential centered in the zero, we have that, for any finite number of couplings in LPA, SSB appears also when it should not (i.e., for $d \leq 2$ and $N \geq 2$ ), and the corresponding (spurious) Wilson-Fisher fixed points lie on the line defined by vanishing mass beta functions. On the other hand using a Taylor expansion of the potential 
around the minimum we can recover Mermin-Wagner theorem even for finite number of couplings.

For the Ising model $(N=1)$ the SSB occurs for $d>2$, again in agreement with exact results. We will also discuss in detail the limit $N \rightarrow \infty$ (the spherical model), where LPA provides exact results for the critical exponents, due to vanishing anomalous dimension (the question of exactness of large $N$ LPA equation was raised in [41] and discussed in detail in [42]).

The paper is organized as follows: after introducing in section 2 the FRG treatment for the $O(N)$ model in dimension $d$, in section 3 we discuss for a general value of $N$ the occurrence of SSB in LPA when a finite number of couplings is used, we discuss the expansion of the effective potential around the zero field; results for the Taylor expansion around the minimum are also presented. The limit $N \rightarrow \infty$ is discussed in section 4 , while an LPA treatment of the appearance of SSB without truncations is presented in section 5. Our conclusions are presented in section 6, while in appendix A we collect useful informations on the regulator functions used in the main text and in appendix B we provide an alternative argument to show the validity of the Mermin-Wagner theorem at LPA level.

\section{Functional renormalization group for the $O(N)$ model}

In the framework of the Kadanoff-Wilson RG approach [15] the differential RG transformations are realized via a blocking construction consisting in the successive elimination of the degrees of freedom which lie above the running momentum cutoff $k$. Consequently, the effective theory defined by the blocked action contains quantum fluctuations whose frequencies are smaller than the momentum cutoff [43-45]. This procedure generates the functional RG flow equation (Wetterich equation) for the effective action $\Gamma_{k}[\phi]$ :

$$
k \partial_{k} \Gamma_{k}[\phi]=\frac{1}{2} \operatorname{Tr}\left[k \partial_{k} R_{k} /\left(\Gamma_{k}^{(2)}[\phi]+R_{k}\right)\right],
$$

where $\Gamma_{k}^{(2)}[\phi]$ denotes the second functional derivative of the effective action. $R_{k}$ is a properly chosen infrared (IR) regulator function which fulfills a few basic constraints to ensure that $\Gamma_{k}$ approaches the bare action in the UV limit $(k \rightarrow \Lambda)$ and the full quantum effective action in the IR limit $(k \rightarrow 0)$ : details are reported in appendix A, where we also discuss the more commonly used regulators for $O(N)$ model and a more general choice able to recover all major types of regulators used in literature [46]. Since RG equations are functional partial differential equations, it is not possible to solve them in general and approximations are required. The approximated RG flow depends on the choice of the regulator function $R$ and the physical results could become scheme-dependent.

One of the commonly used systematic approximations is the truncated derivative expansion where the effective action is expanded in powers of the derivative of the field

$$
\Gamma_{k}[\phi]=\int_{x}\left[U_{k}(\phi)+Z_{k}(\phi) \frac{1}{2}\left(\partial_{\mu} \phi\right)^{2}+\ldots\right] .
$$

In LPA, the higher derivative terms are neglected and the wave-function renormalization is set equal to a constant, i.e. $Z_{k} \equiv 1$. The solution of the RG equations sometimes 
requires further approximations: e.g., in case of the $O(N)$ symmetric scalar field theory the potential can be expanded in powers of the field variable around zero (with a truncation at the power $\left.N_{\mathrm{CUT}}\right)$.

By using the dimensionless potential, $u_{k} \equiv k^{-d} U_{k}$, and dimensionless variables, the Taylor expansion of the potential around zero reads as

$$
u_{k}(\phi)=\sum_{n=1}^{N_{\mathrm{CUT}}} \frac{1}{(2 n) !} g_{n}(k) \phi^{2 n} .
$$

It is convenient to introduce a field variable $\rho=(1 / 2) \phi^{2}$ then the Taylor expanded potential reads as

$$
u_{k}(\rho)=\sum_{n=1}^{N_{\mathrm{CUT}}} \frac{1}{n !} \lambda_{n}(k) \rho^{n} .
$$

As we can see the scale-dependence is encoded in the dimensionless coupling constants, which are related to each other as $g_{n}(k) /(2 n-1) ! !=\lambda_{n}(k)$.

In LPA one obtains the following flow equation for the effective potential for the $d$ dimensional $O(N)$ model

$$
\begin{aligned}
\partial_{t} u & =(d-2) \rho u^{\prime}-d u+\frac{(N-1) A_{d}}{1+u^{\prime}}+\frac{A_{d}}{1+u^{\prime}+2 \rho u^{\prime \prime}} \\
A_{d} & =\frac{1}{2^{d+1}} \frac{1}{\pi^{d / 2}} \frac{1}{\Gamma(d / 2)} \frac{4}{d}:
\end{aligned}
$$

in (2.3) dimensionless variables have been used and $\partial_{t}=k \partial_{k}, u^{\prime}=\partial_{\rho} u, \Gamma(x)$ is the gamma function and for the sake of simplicity here we applied the Litim regulator (as can be seen from equation (A.3a) with $b=1)$.

\section{The truncated $O(N)$ model $\left(N<\infty, N_{\text {CUT }}<\infty\right)$}

Truncation around the zero field. Let us first show the existence of SSB in the expanded $\mathrm{O}(\mathrm{N})$ model with finite $N_{\text {CUT }}$ and finite $N$.

Let consider to start with the simplest case: two couplings $\left(N_{\mathrm{CUT}}=2\right)$ for the Ising case $(N=1)$ in $d=1$. The RG flow equations for the couplings can be derived from (2.3) and reads in this case

$$
\begin{aligned}
\partial_{t} g_{1} & =-2 g_{1}-\frac{1}{\pi} \frac{g_{2}}{\left(1+g_{1}\right)^{2}} \\
\partial_{t} g_{2} & =-3 g_{2}+\frac{6}{\pi} \frac{g_{2}^{2}}{\left(1+g_{1}\right)^{3}}
\end{aligned}
$$

which is obtained by using the Litim regulator. Similar equations are obtained for general $N, d$ and $N_{\mathrm{CUT}}$ (not reported here). In figure 1 the RG flow diagram obtained from (3.1) for the $O(N=1)$ model with two couplings $\left(N_{\mathrm{CUT}}=2\right)$ for $d=1$. The model does not have any phase transition at finite temperature [11], however a Wilson-Fisher (WF) fixed point is clearly visible in figure 1. 


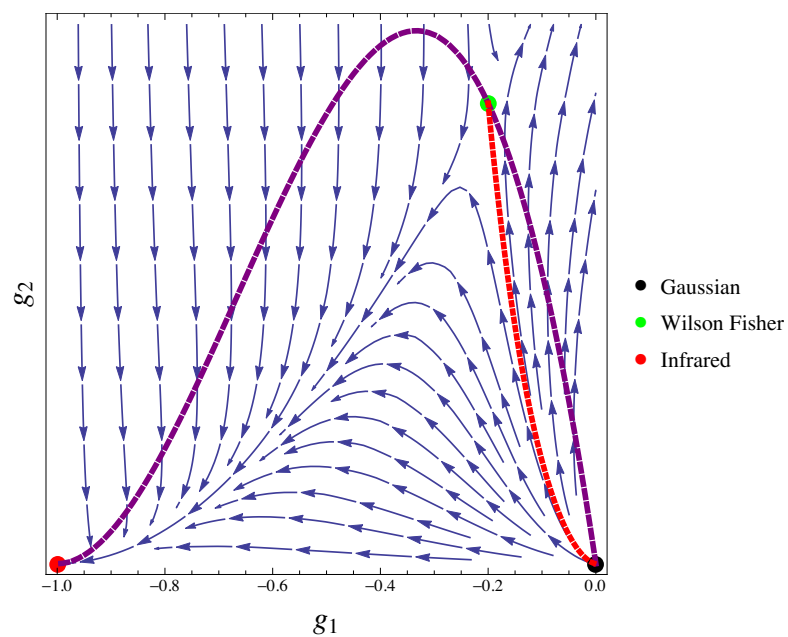

Figure 1. Phase diagram of the $O(N=1)$ model for $d=1$ dimensions obtained by the numerical solution of the RG equations for two dimensionless couplings $\left(N_{\mathrm{CUT}}=2\right)$ using the Litim regulator. Arrows indicate the direction of the flow. The red (dotted) line shows the separatrix and the purple (dashed) line stands for the vanishing mass beta function curve. The Gaussian (black), the WilsonFisher (green) and the IR convexity (red) fixed points are also shown.

The important point we want to stress is that flow diagrams similar to the one depicted in figure 1 can be obtained for any finite $N$ and any finite $N_{\text {CUT }}$ for dimensions $1 \leq d<4$ (by using any regulator functions). In $d=4$, the WF fixed point (green dot in figure 1) merges to the Gaussian one (black). The IR fixed point (red) appears in any dimensions and is related to the convexity of the potential [47-52].

Although one finds similar flows for $d \leq 2$ and $d>2$, there is of course an important difference between the two cases. For $d \leq 2$ the appearance of SSB is not allowed by the Mermin-Wagner theorem, but figure 1 clearly signals the presence of SSB: the red curve from the Gaussian to the WF fixed points separates the phases and the RG trajectories run to the IR fixed point corresponds to the symmetry broken phase. (More comments on the $N=1$ will be given in the following.)

The WF fixed point is situated on the dashed purple line in figure 1 which is determined by the vanishing mass beta function (to which we will refer as the VMB curve). Indeed, from (3.1) one finds

$$
g_{2}=-2 \pi g_{1}\left(1+g_{1}\right)^{2}
$$

which depends on $g_{1}$ and does not depend on higher order couplings even if $N_{\text {CUT }}$ is increased. As a consequence, the VMB curve on the $g_{1}, g_{2}$ plane remains unchanged for any finite value of $N_{\mathrm{CUT}}$. Another important observation is that any fixed point should be situated on the VMB curve (by definition). The role of the VMB curve at LPA level was recently discussed in [53] in connection to the FRG determination of the central charge in $d=2$.

The position of the WF fixed point on the VMB curve depends on $N_{\text {CUT }}$. Similar VMB curves can be drawn for any regulator function with the same properties, as shown in figure 2 by projecting on the $\left(g_{1}, g_{2}\right)$-plane and applying the so called Litim-like regulator 


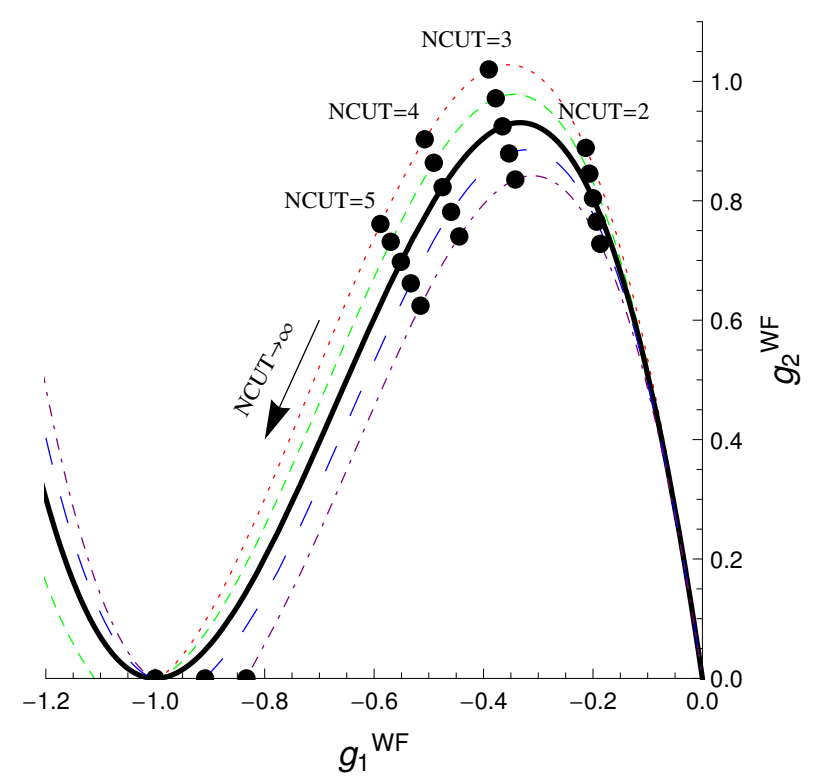

Figure 2. Position of the WF fixed point on the VMB curves for the $O(N=1)$ model in $d=1$ for various values of $N_{\mathrm{CUT}}$. Different lines correspond to different regulators, i.e. $0.8<h<1.2$ is chosen in (3.4). The solid line corresponds to $h=1$, i.e. to the Litim regulator. The IR fixed point remains unchanged if $h \leq 1$.

class. Using the notation of appendix A, this regulator class corresponds to various values of the parameter $h$ but with $b=1$ ( $h=1, b=1$ corresponds the Litim regulator):

$$
r=\left(\frac{1}{y}-h\right) \Theta(1-h y)
$$

(the regulator (3.4) is obtained by taking the $c \rightarrow 0$ limit in (A.2)).

We observe that the position of the Gaussian fixed point is scheme-independent and thus the VMB curves always start form zero and go through the scheme-dependent IR fixed point.

We found and verified that plots qualitatively similar to figure 2 are obtained for general $N$ and $1 \leq d \leq 2$ (with $\mathrm{d}$ real): in these cases the WF fixed points corresponding to the different regulators for increasing $N_{\mathrm{CUT}}$ tend to the (respective) IR fixed points. This has to be contrasted with the situation in $d=3$ (or more generally for real $d \geq 2$ ), as it is shown in figure 3: namely, for increasing values of $N_{\mathrm{CUT}}$ the WF fixed points does not tend to the IR ones as for $d=1$, but tend to constant (non-trivial) WF fixed points for $d=3$, as indicated in figure 3. These non-trivial WF fixed points can be computed for $N_{\text {CUT }} \rightarrow \infty$, i.e. treating exactly LPA, using the spike plot method [56, 57]. Clearly, the position of these non-trivial WF fixed points depend on the choice of the regulator.

Again, the scenario presented in figure 3 is the same to what happens for general $N$ and $2<d<4$. In order to illustrate this we plot the dependence of $g_{1}$ (figure 4 ) and $g_{2}$ (figure 5) on the truncation parameter $N_{\mathrm{CUT}}$ for $d=1$ and $d=3$ for three different values of $N$. 


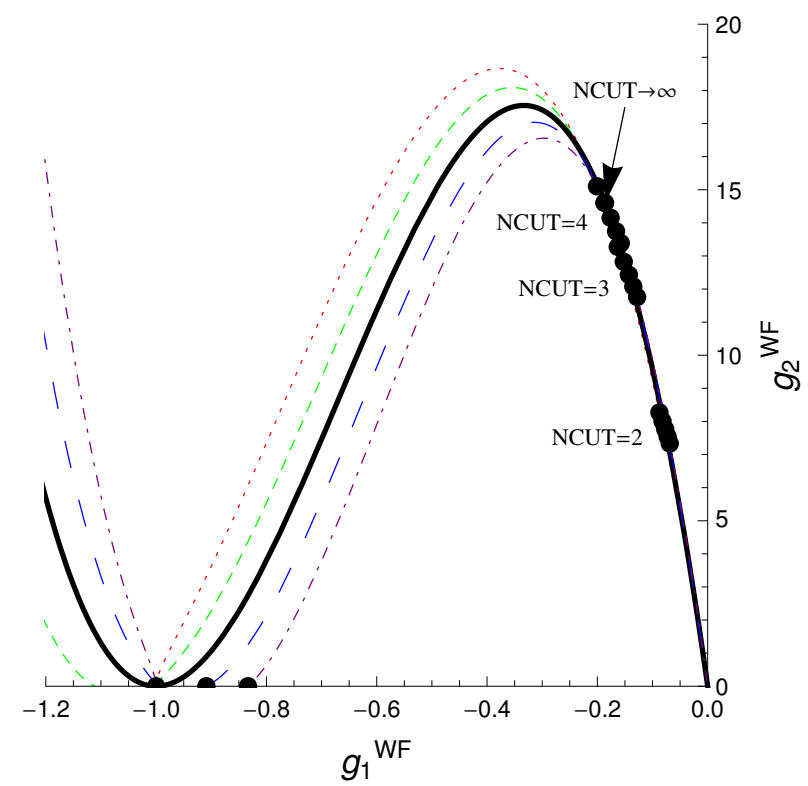

Figure 3. Position of the WF fixed point on the VMB curves for the $O(N=1)$ model in $d=3$ for various values of $N_{\mathrm{CUT}}$. Different lines correspond to different regulators, i.e. $0.8<h<1.2$ is chosen in (3.4) as in figure 2. The $N_{\mathrm{CUT}} \rightarrow \infty \mathrm{WF}$ fixed point (shown for the Litim regulator) is computed using the spike plot method $[56,57]$.

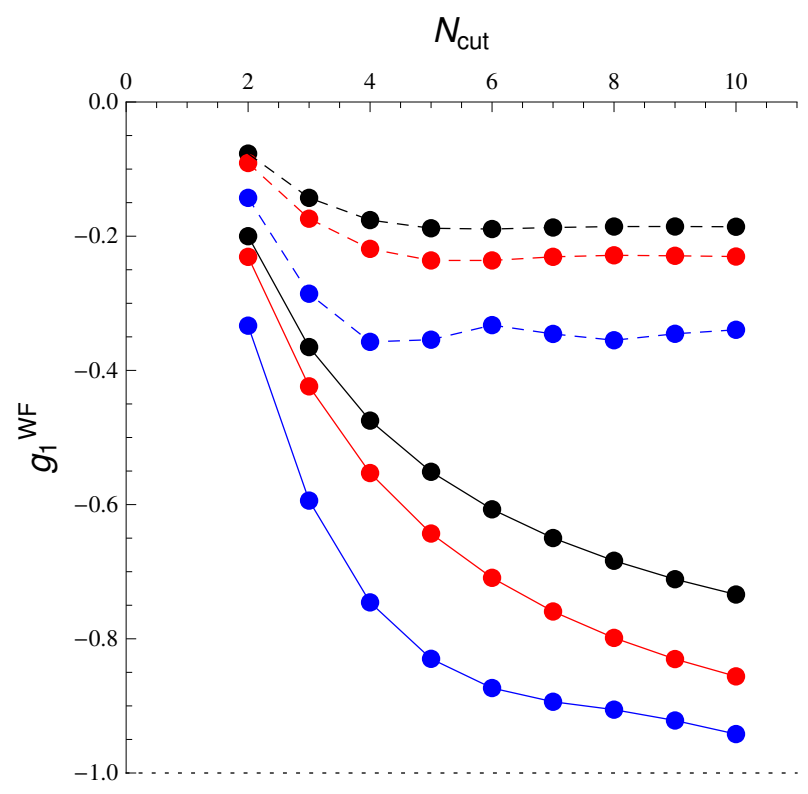

Figure 4. The $g_{1}$ coordinate of the WF fixed point of the $O(N)$ model is shown as a function of $N_{\text {CUT }}$ for $N=1$ (black), $N=2$ (red) and $N=10$ (blue) from top to bottom for $d=1$ (solid lines) and for $d=3$ (dashed lines). 


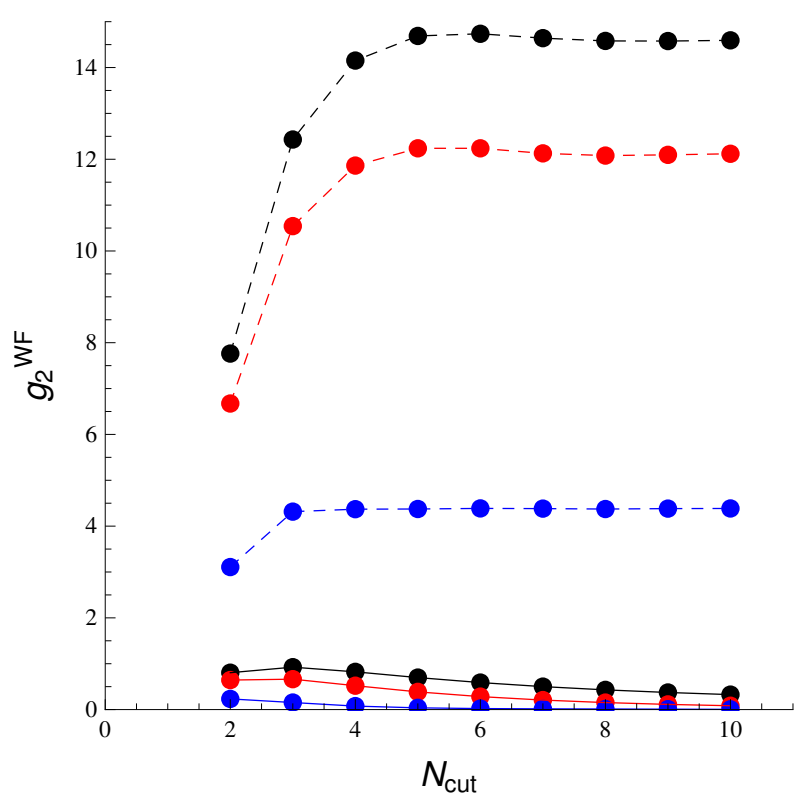

Figure 5. The $g_{2}$ coordinate of the WF fixed point of the $O(N)$ model as a function of $N_{\mathrm{CUT}}$ : as in figure 4 from top to bottom it is $N=1$ (black), $N=2$ (red) and $N=10$ (blue) for $d=1$ (solid lines) and for $d=3$ (dashed lines).

In summary, the results obtained for the $O(N)$ model with finite $N$ and finite $N_{\text {CUT }}$ show the existence of SSB and a WF fixed point (distinct from the Gaussian one) for $1 \leq d<4$ (with $d$ real), but indicated that for $N_{\mathrm{CUT}} \rightarrow \infty$ the symmetry broken phase disappears (persists) for $d \leq 2(d>2)$. This result has to be verified without operating the truncation of the couplings, i.e. treating exactly the LPA equation: we may refer to this as to the "non-truncated" $O(N)$ model to emphasize the fact that at LPA level no approximation is done. For finite $N$ this will be done in section 5 , while the next section is devoted to the spherical model limit $N \rightarrow \infty$.

Truncation around the minimum. Now we will consider a Taylor expansion of the effective potential around the minimum,

$$
u_{k}(\rho)=\sum_{i=2}^{N_{\mathrm{CUT}, \mathrm{m}}} \frac{\lambda_{k, i}}{i !}\left(\rho-\rho_{0}\right)^{i},
$$

where $N_{\text {CUT,m }}$ is the truncation number around the minimum. In this case the results are drastically different. First of all we will consider this truncation at the minimum level $N_{\mathrm{CUT}, \mathrm{m}}=2$. Obviously it is possible to relate the values of the coupling defined around the zero $g_{1}$ and $g_{2}$ with the values of the coupling $\lambda$ and the running minimum $\rho_{0}$. However this relations, which give the correct result for the WF fixed point, are not working for the Gaussian fixed point, which is $g_{1}=g_{2}=0$, but no solution of the fixed point equations for $\rho_{0}$ and $\lambda$ has a vanishing $\rho_{0}$.

The truncation around the minimum includes just one running coupling $\lambda_{k, 2} \equiv \lambda$ and the running minimum value $\rho_{0}$. From equation (2.3) we can obtain flow equations for these 
two quantities [20,21], we report them for general real dimension $d$ and $N$,

$$
\begin{aligned}
\partial_{t} \rho_{0} & =(d-2) \rho_{0}+A_{d}\left(1-N-\frac{3}{\left(1+2 \rho_{0} \lambda\right)^{2}}\right) \\
\partial_{t} \lambda & =\lambda\left(4-d-2 A_{d}\left(N-1+\frac{18 \lambda}{\left(1+2 \rho_{0} \lambda\right)^{3}}\right)\right) .
\end{aligned}
$$

One can look for the fixed point solutions for $\rho_{0}$ and $\lambda$. In the particular case of the Ising model $(N=1)$ one gets, for example, the Wilson-Fisher fixed point given by

$$
\begin{aligned}
\rho_{0} & =\frac{4(2 d-5)^{2} A_{d}}{3(d-2)^{3}}, \\
\lambda & =\frac{3(4-d)(d-2)^{3}}{16(2 d-5)^{3} A_{d}} .
\end{aligned}
$$

The corresponding expression for $N>1$ are very lengthy and are not reported here.

From the solution (3.8)-(3.9) one sees that the value of the minimum $\rho_{0}$ is well defined (positive) for every value of $d$ as long as $d>2$ : a similar result is valid for every $N$.

For $d>4$ the solution for $\lambda$ is negative, and, again, this is true for every $N$. Also it should be noted that the coupling $\lambda$, for $N=1$, is diverging at $d=2.5$ and turns out to be negative for $d<2.5$, thus giving an unphysical solution for $d<2.5$ : we know that this is not true, since in $d=2$ for $N=1$ there is SSB. This is not valid in the general $N$ case where the coupling $\lambda$ has only a maximum and is not diverging at $d=2.5$ and no sign change is present at any value of $d>2$.

In figure 6 is reported the minimum value $\rho_{0}$ as a function of the dimension at the WF fixed point for various $N$ values, the minimum diverges for $d=2$ for every $N$ in agreement with the Mermin-Wagner theorem. In the inset the values for the coupling $\lambda$ are shown. The coupling stays finite and positive for $d>2$ and $N>1$, but for $N=1$ it is diverging at $d=2.5$ and turning negative at $d<2.5$, then this truncation is not giving a reliable lower critical dimension for the Ising model.

We expect that larger values of $N_{\mathrm{CUT}, \mathrm{m}}$ do not to change the main qualitative results just presented.

This truncation, while giving the correct behavior for the SSB, cannot catch the noninteracting (Gaussian) fixed point and thus gives only a partial description of the theory phase space. In the next sections we will show how it is possible to reproduce some of the correct result retrieved here and to go beyond them with a simple analysis of the exact flow equation for the effective potential (2.3).

\section{The spherical model without truncations $\left(N=\infty, N_{\mathrm{CUT}}=\infty\right)$}

In this section we consider the $O(N)$ model in the large $N$ limit: thus we can neglect the terms in (2.3) which are in the order of $1 / N$. To see this we are going to rescale $(2.3)$ by a parameter $\left(A_{d} N\right)$, and considering the new variables $\rho \rightarrow \rho /\left(A_{d} N\right)$ and $u \rightarrow u /\left(A_{d} N\right)$. The derivative of the potential remains invariant under this rescaling $u^{\prime} \rightarrow \frac{\partial u /\left(A_{d} N\right)}{\partial \rho /\left(A_{d} N\right)}=u^{\prime}$. As a 


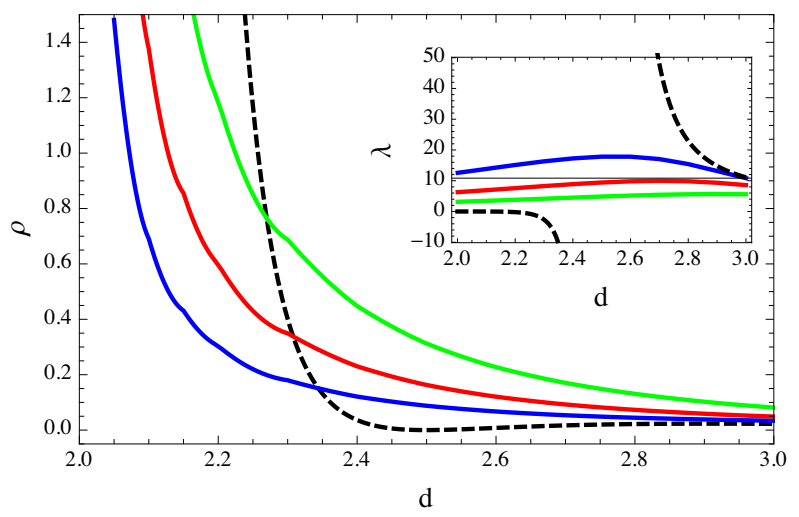

Figure 6. Running minimum (main plot) and coupling (inset) values for the WF fixed point in the truncation around the minimum at $N_{\mathrm{CUT}, \mathrm{m}}=2$ as a function of the dimension $d$, for the Ising model ( $N=1$ black dashed curves), the XY model $(N=2$, blue solid curves), the Heisenberg model ( $N=3$, red solid curves) and the $N=5$ model (green solid curves). The Ising coupling $\lambda$ is the only one which is diverging and then turning negative at $d=2.5$ this is in contrast both with the well known exact solution of the Ising model in $d=2$ and with the following argument on exact solutions of equation (2.3).

first step, we divide the RG equations (2.3) by $A_{d} N$ keeping the potential non-truncated $\left(N_{\mathrm{CUT}}=\infty\right)$ : one finds

$$
\partial_{t} \frac{u}{A_{d} N}=(d-2) \frac{\rho}{A_{d} N} u^{\prime}-d \frac{u}{A_{d} N}+\frac{1}{1+u^{\prime}}-\frac{1}{N} \frac{1}{1+u^{\prime}}+\frac{1}{N} \frac{1}{1+u^{\prime}+2 \rho u^{\prime \prime}} .
$$

Next we perform the rescaling

$$
\partial_{t} u=(d-2) \rho u^{\prime}-d u+\frac{1}{1+u^{\prime}}-\frac{1}{N} \frac{1}{1+u^{\prime}}+\frac{1}{N} \frac{1}{1+u^{\prime}+2 \rho u^{\prime \prime}} .
$$

By taking the limit $N \rightarrow \infty$ the following terms remain:

$$
\partial_{t} u=(d-2) \rho u^{\prime}-d u+\frac{1}{1+u^{\prime}} .
$$

This simplified expression represents the RG equation for the effective potential of the large $N O(N)$ model in arbitrary dimension. From the equation (4.3) we can extract some useful information. First we should differentiate it by $\rho$ in order to get an equation for the derivative of the potential. It reads then

$$
\partial_{t} u^{\prime}=(d-2) u^{\prime}+(d-2) \rho u^{\prime \prime}-d u^{\prime}-\frac{u^{\prime \prime}}{\left(1+u^{\prime}\right)^{2}} .
$$

Since in a physically reasonable theory the potential is bounded from below, we can assume that this potential has a global minimum at some $\rho=\rho_{0}$. For $\rho_{0}$ we have the following value for the derivatives of the potential at the fixed point: $u^{\prime}\left(\rho_{0}\right)=0, u^{\prime \prime}\left(\rho_{0}\right) \equiv \lambda$. Assuming that the quartic coupling $\lambda$ is finite, we have then the following equation:

$$
0=(d-2) \rho_{0} \lambda-\lambda
$$


with the solution

$$
\rho_{0}=\frac{1}{d-2}
$$

which determines the cases where the minimum of the potential can be found or not in the large $N$ case. There is SSB if the potential has the minimum at some finite $\rho_{0}>0$ : in the case of (4.6) we can satisfy this condition for $d>2$. For $d<2$ we find $\rho_{0}<0$, hence there will be no SSB. The $d=2$ case seems to be undefined, since $\rho_{0} \rightarrow \infty$ in this limit. However, if the minimum of the potential is sent to infinity one cannot define a proper minimum. The absence of a finite minimum indicates the absence of the spontaneous symmetry breaking for $d=2$ dimensions. This can be also seen by solving eq. (4.4) using the method of characteristics. ${ }^{1}$ The large $N$ limit is a frequently used technique [54] where the results obtained can be considered as exact ones since the LPA approximation becomes exact when $N \rightarrow \infty$ [55].

\section{The $O(N)$ model without truncations $\left(N<\infty, N_{\mathrm{CUT}}=\infty\right)$}

In this section we finally consider the problem of determining the lower critical dimension for the $O(N)$ model for a finite $N$ but keeping the potential non-truncated in LPA.

Let us first consider the $N=1$ case by trying the following strategy: numerically calculate the WF fixed point position for finite $N_{\mathrm{CUT}}$ and approximate the limit $N_{\mathrm{CUT}} \rightarrow$ $\infty$. Notice that, without knowing the exact WF fixed point positions, it is difficult for dimensions around $d=2$ to unambiguously extract from the limit of increasing $N_{\text {CUT }}$ the value of the non-truncated model. For this reason we determine the WF fixed point for $N_{\mathrm{CUT}}=\infty$ by using the spike plot method $[56,57]$ in LPA.

The (finite $N_{\text {CUT }}$ ) results are plotted in figure 7 where we show that the $N_{\text {CUT }}$ dependence of the WF fixed points on the VMB curves (obtained for Litim regulator) depends on the value of the dimension $d$ : several $d$ between $d=1$ and $d=3$ (including $d=2$ ) are plotted for $N=1$. Similar plots are obtained for general value of $N$. The positions of the exact WF fixed points, computed by the spike plot method, are also indicated for each case (by the symbol $X$ ).

Figure 7 clearly shows that for $N_{\mathrm{CUT}} \rightarrow \infty$ the $g_{2}$ coordinate of the WF fixed point tends to a finite value for $d>2$ and runs to zero for $d \leq 2$ : since this property is found to be valid in LPA for general values of $N$, when applied to $N \geq 2$ this result implies that the LPA is enough to reproduce the content of the Mermin-Wagner theorem. For $d=2$ (sixth line from top in figure 7) one finds from the spike plot analysis that (for all $N$ ) no WF fixed point occurs: this result is correct for $N \geq 2$, but not for the Ising model $(N=1)$. As we will later discuss, for the Ising model in $d=2$ one needs to apply $\mathrm{LPA}^{\prime}$ approximation.

Given the clear numerical evidence that Mermin-Wagner is well obtained in the limit of increasing $N_{\mathrm{CUT}}$ and the excellent agreement with the spike plot method findings for the WF fixed points, we investigated and present in the following two analytical arguments valid for the non-truncated (exactly treated) LPA confirming these results.

\footnotetext{
${ }^{1}$ We acknowledge J.M. Pawlowski for discussions on this point.
} 


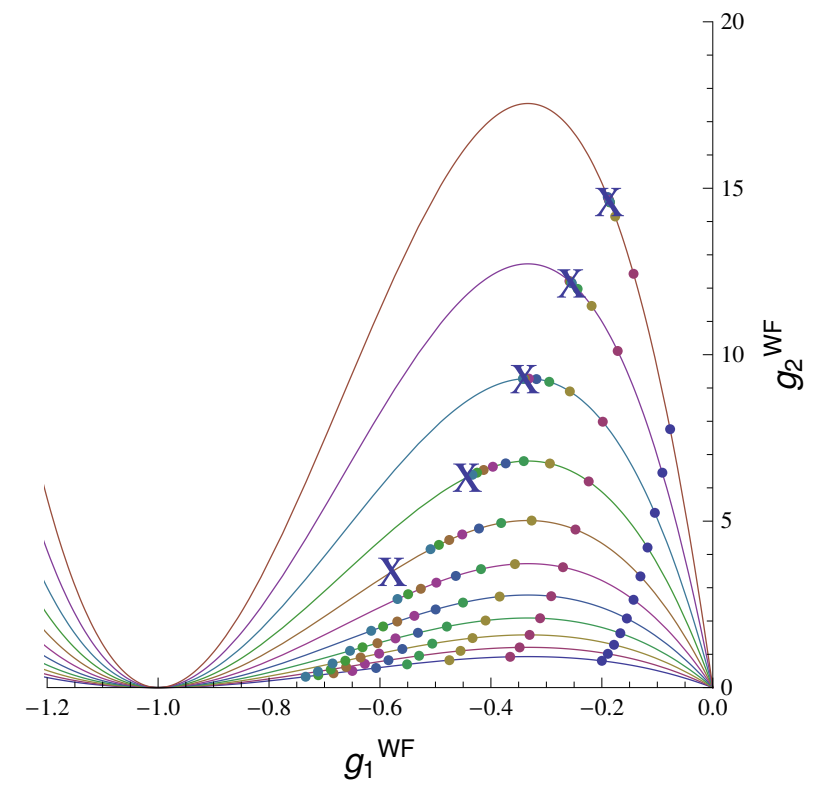

Figure 7. The figure shows the positions of the WF fixed points and the corresponding VMB curves of the $O(N=1)$ model for various values of $N_{\mathrm{CUT}}$ for 11 different values of the dimension $1 \leq d \leq 3$ having the values $d=3,2.8,2.6,2.4,2.2,2,1.8,1.6,1.4,1.2,1$. The VMB curves obtained for these dimensions are plotted from the top to the bottom in decreasing order. For each value of $d$, from right one has $N_{\mathrm{CUT}}=2, \cdots, 10$. The exact WF points are indicated by the symbol $X$ and are obtained by the spike plot method $[56,57]$.

In order to consider the appearance of SSB for the $O(N)$ model for finite $N$ but keeping the potential unexpanded $\left(N_{\mathrm{CUT}}=\infty\right)$, let us start with the fixed point equation of $(2.3)$ : putting $\partial_{t} u=0$ one has

$$
d u-(d-2) \rho u^{\prime}=\frac{A_{d}(N-1)}{1+u^{\prime}}+\frac{A_{d}}{1+u^{\prime}+2 \rho u^{\prime \prime}} .
$$

The 1.h.s. of the RG equation (5.1) is linear in the effective potential. The r.h.s. depends on the effective potential and its derivatives non-linearly, thus we introduce the notation

$$
\begin{aligned}
L P & \equiv d u(\rho)-(d-2) \rho u^{\prime}(\rho), \\
N L P & \equiv \frac{A_{d}(N-1)}{1+u^{\prime}(\rho)}+\frac{A_{d}}{1+u^{\prime}(\rho)+2 \rho u^{\prime \prime}(\rho)},
\end{aligned}
$$

where $L P(N L P)$ stands for the linear (non-linear) part. Let us consider the large field limit $(\rho \gg 1$ ) of equation (5.1). First of all we assume analyticity of the effective potential [58] at any finite value of the field, then the potential at infinity can only either be a constant or divergent. In the first case $N L P$ is constant at infinity and the potential will be just a constant at any $\rho$. In the second case $N L P$ in principle may vanish or tend to a constant (eventually zero) at infinity, thus the fixed point potential $u(\rho)$ must satisfy for large field the condition

$$
L P=C,
$$


where $C$ is a finite (or zero) constant to be consistently determined. Then one finds the solution for $\rho \gg 1$

$$
u(\rho)=\frac{C}{d}+a \rho^{\frac{d}{d-2}},
$$

where $a$ is a proportionality constant. Now let us differentiate the previous expression of $u(\rho)$ : this yields $u^{\prime}(\rho)=a \frac{d}{d-2} \rho^{\frac{2}{d-2}}$, which for large $\rho$ gives a diverging quantity for $d>2$ and a zero for $d<2$. In the former case we are violating the assumption that $u^{\prime}$ is bounded for large $\rho$, while in the latter the constant $C$ is zero. In both cases we find

$$
u(\rho)=a \rho^{\frac{d}{d-2}}
$$

for $\rho \rightarrow \infty$,

The general solution of equation (5.1), which is not a constant, can be then divided into two parts,

$$
u(\rho)=f(\rho)+a \rho^{\frac{d}{d-2}},
$$

where the function $f(\rho)$ is subjected to the condition

$$
\lim _{\rho \rightarrow \infty} f(\rho)=0 .
$$

The physical Gibbs free energy $F(m)$ can be computed from the effective potential $u(\rho)$ passing from dimensionless variables to the dimensional ones [41]: one finds

$$
F(m)=k^{d} u\left(k^{2-d} m^{2}\right)=k^{d} f\left(k^{2-d} m^{2}\right)+a m^{\frac{2 d}{d-2}},
$$

where $m$ is the dimensional field of our model, which in the case of a spin system is the average magnetic moment. The free energy of the system is obtained then in the $k \rightarrow 0$ limit of equation (5.8), where we should distinguish between three cases.

\section{$5.1 d>2$}

When $d>2$ the factor $k^{2-d}$ in the argument of the function $f(\rho)$, in eq. (5.8) is diverging. However we know from the analysis of the general solution that the function $f(\rho)$ tends to a constant in the infinite limit of its argument, hence the Gibbs free energy for an $O(N)$ model in LPA for $d>2$ reads

$$
a m^{\frac{2 d}{d-2}}
$$

where $a$ is positive and can be fixed following the procedure described in [58].

\section{$5.2 d<2$}

In the case $d<2$ the situation drastically changes. Indeed, the factor $k^{2-d}$ in the argument of $f(\rho)$ in equation (5.8) is now vanishing. The behaviour of the $f(\rho)$ function for vanishing argument can be obtained from equation (5.7).

Since we know that such expression should be defined for any finite value of field $\rho[58]$, then $f(\rho)$ is diverging in zero, in order to compensate the divergence of $\rho^{\frac{d}{d-2}}$, which 
has a negative exponent in $d<2$. Thus the behaviour of $f(\rho)$ in the limit of vanishing arguments is

$$
\lim _{x \rightarrow 0} f(x)=w(x)-a x^{\frac{d}{d-2}},
$$

where $w(x)$ is finite in zero. Substituting the last expression into eq. (5.8) one obtains

$$
k^{d} w\left(k^{2-d} m^{2}\right),
$$

which is zero in the $k \rightarrow 0$ limit.

In summary, we obtained that for $d>2$ the critical free energy of a $O(N)$ model can have the form (5.9) or can be zero, thus the phase transition is present [58]. For $d<2$ the fixed point free energy can only be zero and no spontaneous symmetry breaking is allowed. In appendix B we report an alternative derivation of this result, again based on the analysis of LPA equation.

\section{$5.3 d=2$}

The previous argument cannot be directly used for $d=2$. The numerical study of the equation (5.1), i.e. $L P=N L P$, reveals in this case that for $N \geq 2$ the LPA gives the Mermin-Wagner result: indeed the solution for large field of $u(\rho)$ turns out to be oscillatory, therefore correctly implying the absence of SSB and the Mermin-Wagner theorem. However, such oscillatory solutions persist for $N=1$, predicting the absence of SSB, which is clearly wrong. A derivation of the fact that in LPA without truncations SSB does not occur in $d=2$ at LPA level is provided in appendix B.

To have a complete picture of the $d=2$ case one should go beyond LPA: as discussed in [10], in LPA' the limit of the anomalous dimension $\eta$ for $d \rightarrow 2$ is vanishing provided that $N \geq 2$, and non-vanishing for $N=1$. This gives a clear explanation in the FRG framework of the presence or the lack of SSB in $O(N)$ models in $d=2$. Of course, the fact that there is no SSB for $N=2$ does not imply the absence of the Berezinskii Kosterlitz-Thouless transition [59, 60], as can be seen also in FRG treatments [10, 61-63].

\section{Conclusions}

In this paper we studied spontaneous symmetry breaking (SSB) for $O(N)$ models using functional renormalization group (FRG) techniques, showing that even the local potential approximation (LPA) when treated without further approximations is sufficient to give qualitatively correct results. For systems with continuous symmetry $(N \geq 2)$ LPA gives no SSB for $d \leq 2$ and SSB for $d>2$ in agreement with the Mermin-Wagner theorem and its extension to systems with fractional dimension; in particular, simple analytical expressions are found in the large $N$ limit, correctly retrieving the expected results for the spherical model. We observe that the presented results rule out any type of SSB, not only the standard (bicritical) Wilson-Fisher (WF) fixed point, but also all the other possible multicritical fixed points.

As a tool to assess the validity of different truncation schemes, for general $N$ we studied the solutions of the LPA renormalization group equations using a finite number of terms (and different regulators), showing that SSB always occurs even where it should not (i.e. for $d \leq 2$ for $N \geq 2$ ). The SSB is signalled by WF fixed points which for any possible truncation 
are shown to stay on the line defined by vanishing mass beta functions. Increasing the number of couplings these WF fixed points tend to the infrared convexity fixed point for $d \leq 2$ and to the pertinent exact LPA WF point for $d>2$. Moreover we studied the case of Taylor expansion of the effective potential around the minimum $\rho_{0}$. Even when this expansion is truncated at lowest order $N_{\mathrm{CUT}, \mathrm{m}}=2$, it is possible to retrieve the correct behavior for the Mermin-Wagner theorem, since $\rho_{0}$ is diverging when $d \rightarrow 2$. However at this order the truncation around the minimum cannot provide the expected behavior for the $N=1$ case, since the coupling $\lambda$ diverges at $d=2.5$ and becomes negative below this threshold, even if it is well known that in the Ising model the SSB occurs even at $d=2$.

For the Ising model $(N=1)$ the SSB is shown to occur for $d>2$ (as it should be), but not for $d=2$ (as it should not be). At variance, finding the correct results for $d=2$ and $N=1$, as well as for the Ising model in $1<d<2$, requires to go beyond LPA since the anomalous dimension cannot be neglected: in $d=2$ the LPA without truncations is sufficient to explain the absence of SSB for $N \geq 2$, but not to predict the presence of SSB for the Ising model. To have qualitatively correct results in $d=2$ valid for all $N$ anomalous dimension effects as introduced in $\mathrm{LPA}^{\prime}$ have to be considered. This has been recently shown in [10], which shows how LPA' $^{\prime}$ is able to reproduce numerically the behaviour predicted by the Mermin-Wagner theorem for $d \rightarrow 2$ and $N \geq 2$ (with the anomalous dimension $\eta \rightarrow 0$ and the correlation length exponent $\nu \rightarrow \infty$ [53]), and correctly predicting at the same time SSB and a finite anomalous dimension exponent for the Ising model. We extended these results showing that when the anomalous dimension vanishes then no SSB transition is possible in $d \leq 2$ (as it happens for the $O(N \geq 2)$ models). Motivated by these findings, a study based on FRG of the Ising model in dimensions smaller than 2 is in our opinion worthwhile of future work.

\section{A Regulator functions}

Regulator functions have already been discussed in the literature by introducing their dimensionless form

$$
R_{k}(p)=p^{2} r(y), \quad y=p^{2} / k^{2},
$$

where $r(y)$ is dimensionless. Various types of regulator functions can be chosen, but a more general choice is the so called CSS regulator [46] which recovers all major types of regulators in its appropriate limits. By using a particular normalization $[64,65]$ it has the following form

$$
r_{\mathrm{css}}^{\mathrm{norm}}(y)=\frac{\exp [\ln (2) c]-1}{\exp \left[\frac{\ln (2) c y^{b}}{1-h y^{b}}\right]-1} \theta\left(1-h y^{b}\right),
$$

with the Heaviside step function $\theta(y)$ where the limits are

$$
\begin{aligned}
\lim _{c \rightarrow 0, h \rightarrow 1} r_{\mathrm{css}}^{\mathrm{norm}} & =\left(\frac{1}{y^{b}}-1\right) \theta\left(1-y^{b}\right), \\
\lim _{c \rightarrow 0, h \rightarrow 0} r_{\mathrm{css}}^{\mathrm{norm}} & =\frac{1}{y^{b}}, \\
\lim _{c \rightarrow 1, h \rightarrow 0} r_{\mathrm{css}}^{\mathrm{norm}} & =\frac{1}{\exp \left[\ln (2) y^{b}\right]-1} .
\end{aligned}
$$


Thus, the CSS regulator has indeed the property to recover all major types of regulators: the Litim [66-68], the power-law [45] and the exponential [43, 44] ones.

\section{B Spontaneous symmetry breaking at finite $N$}

In this appendix we provide an alternative derivation of the validity of the Mermin-Wagner theorem in LPA.

The fixed point equation (5.1) for the potential, using the notation (5.2)-(5.3), reads $L P=N L P$. In the large field limit $(\rho \rightarrow \infty)$ the potential could be diverging or bounded, hence tending to a constant value. Let us consider in detail the second case: $N L P$ is then converging to a constant, since $u^{\prime}$ and $u^{\prime \prime}$ vanish and the following differential equation is found

$$
d u(\rho)-(d-2) \rho u^{\prime}(\rho)=c .
$$

The solution for this equation is

$$
u(\rho)=\frac{c}{d}+a \rho^{\frac{d}{d-2}} .
$$

Here, $c$ is the constant representing the large field limit of the $N L P$ and $a$ is another constant, obtained from the integration. Considering our assumption on $u$ in the $\rho \rightarrow \infty$ limit, namely that it is a constant, the constant of the integration can take only one value: $a=0$. It follows that $u=c / d=$ constant (the asymptotics).

Let us now consider the case when $u(\rho)$ is diverging in the large field limit. In this case we need to distinguish three sub-cases considering the behaviour of the derivative, $u^{\prime}(\rho)$, since it can be diverging, tending to a finite value or to zero. In the last two cases $N L P$ tends towards a constant again. So the differential equation which should be solved has the same form as (B.1). Hence the solution is again (B.2). In the case when $u^{\prime}$ is also diverging $N L P$ tend to zero, hence the differential equation slightly modifies:

$$
d u(\rho)-(d-2) \rho u^{\prime}(\rho)=0
$$

yielding the solution:

$$
u(\rho)=a \rho^{\frac{d}{d-2}} .
$$

Now we can consider the constant of integration $a$ for each case. Due to the stability requirement of the potential, that is $u$ has to be bounded from below, $a$ is being forced to be a positive real for all the three sub-cases. We can then write the form of the potential in the following as

$$
u(\rho)=g(\rho)+a \rho^{\frac{d}{d-2}}
$$

where, $g(\rho)$ is a constant (or vanishes) in the large $\rho$ limit.

We are looking for the minimum $\rho_{0}$ : let us differentiate equation (B.5) and take it at $\rho=\rho_{0}$, which is assumed to be the minimum. Performing this operation one gets

$$
0=g^{\prime}\left(\rho_{0}\right)+a \frac{d}{d-2} \rho_{0}^{\frac{d}{d-2}-1} .
$$


The minimum can be then expressed as

$$
\rho_{0}=\left(-\frac{g^{\prime}\left(\rho_{0}\right)}{a}\right)^{\frac{d-2}{2}}\left(\frac{d-2}{d}\right)^{\frac{d-2}{2}} .
$$

We can now distinguish three sub-cases:

- for $d>2$ the second factor in the expression of the minimum has a positive real value. We have established already that the in the first factor the denominator $a$ is positive. Therefore $g^{\prime}\left(\rho_{0}\right)$ must be negative or zero in order to fulfill the equation (B.6). Hence altogether the fraction in the bracket must be positive independently from the dimension. So we found that for $d>2$ the $\rho_{0}$ can be either vanishing or finite positive. This indicates the presence of SSB.

- for $d=2$ the second factor gives a $0^{0}$, which is indeterminate, or alternatively one can define it as 1 if we consider the $d=2$ case as a limit $(d \rightarrow 2)$. In this instance what one can see already in the (B.6) is that if we assumed for $\rho_{0}$ to be a positive real, then $g^{\prime}\left(\rho_{0}\right)$ would be $-\infty$ to compensate the second term. Hence (B.7) is undefined or alternatively if we say $d \rightarrow 2$, then $\rho_{0}=\infty$, which means there is no finite positive minimum to consider, therefore no SSB occours in $d=2$ limit. $^{1}$

- for $d<2$ one can immediately see that the second factor in (B.7) is going to have complex value(s). From equation (B.6) we can conclude that $g^{\prime}\left(\rho_{0}\right) \geq 0$ for $d<2$. The only value for $g^{\prime}\left(\rho_{0}\right)$ that makes B.7 physically sensible is when $g^{\prime}\left(\rho_{0}\right)=0$, therefore the potential cannot have a true extremum (minimum) anywhere else than $\rho_{0}=0$. This clearly shows that there exists only a symmetric phase for dimensions $d<2$ in LPA.

For the $u \rightarrow$ constant case we can do essentially a similar argument.

The conclusion is that the Mermin-Wagner theorem can be shown using FRG techniques in the LPA.

\section{Acknowledgments}

We thank A. Codello, G. Gori, E. Marchais, J.M. Pawlowski and D. Zappalà for discussions. We are also grateful to A. Rancon for very useful comments on the effects of the truncation around the minimum. This research was supported by the European Union and the State of Hungary, co-financed by the European Social Fund in the framework of TAMOP-4.2.4.A/ 2-11/1-2012-0001 'National Excellence Program', by the TÁMOP 4.2.1./B-09/1/KONV2010-0007 project and also through the project Supercomputer, the national virtual lab (grant no.: TAMOP-4.2.2.C-11/1/KONV-2012-0010). A.T. acknowledges support from the Italian PRIN "Fenomeni quantistici collettivi: dai sistemi fortemente correlati ai simulatori quantistici" (PRIN 2010_2010LLKJBX). Support from the CNR/MTA Italy-Hungary 2013-2015 Joint Project "Non-perturbative field theory and strongly correlated systems" is gratefully acknowledged. 
Open Access. This article is distributed under the terms of the Creative Commons Attribution License (CC-BY 4.0), which permits any use, distribution and reproduction in any medium, provided the original author(s) and source are credited.

\section{References}

[1] K. Huang, Statistical Mechanics, Wiley, New York (1987).

[2] N.D. Mermin and H. Wagner, Absence of ferromagnetism or antiferromagnetism in one-dimensional or two-dimensional isotropic Heisenberg models, Phys. Rev. Lett. 17 (1966) 1133 [INSPIRE].

[3] P.C. Hohenberg, Existence of Long-Range Order in One and Two Dimensions, Phys. Rev. 158 (1967) 383 [INSPIRE].

[4] S. Coleman, There are no Goldstone bosons in two dimensions, Comm. Math. Phys. 31 (1973) 259 [INSPIRE].

[5] S. Stringari, Sum Rules and Bose-Einstein Condensations, in Bose-Einstein condensation, A. Griffin, D.W. Snoke and S. Stringari eds., Cambridge University Press, Cambridge (1995), p. 86 [cond-mat/9311024].

[6] L.P. Kadanoff, Statistical physics: statics, dynamics and renormalization, World Scientific, Singapore (2000).

[7] D. Cassi, Phase transitions and random walks on graphs: A generalization of the Mermin-Wagner theorem to disordered lattices, fractals, and other discrete structures, Phys. Rev. Lett. 68 (1992) 3631.

[8] D. Cassi, Local vs Average Behavior on Inhomogeneous Structures: Recurrence on the Average and a Further Extension of Mermin-Wagner Theorem on Graphs, Phys. Rev. Lett. 76 (1996) 2941.

[9] R. Burioni and D. Cassi, Universal Properties of Spectral Dimension, Phys. Rev. Lett. 76 (1996) 1091.

[10] A. Codello and G. D'Odorico, $O(N)$-Universality Classes and the Mermin-Wagner Theorem, Phys. Rev. Lett. 110 (2013) 141601 [arXiv:1210.4037] [INSPIRE].

[11] G. Mussardo, Statistical field theory: an introduction to exactly solved models in statistical physics, Oxford University Press, Oxford (2010).

[12] R. Burioni, D. Cassi and A. Vezzani, Inverse Mermin-Wagner theorem for classical spin models on graphs, Phys. Rev. E 60 (1999) 1500.

[13] H.E. Stanley, Dependence of critical properties on dimensionality of spins, Phys. Rev. Lett. 20 (1968) 589 [INSPIRE].

[14] G.S. Joyce, Critical Properties of the Spherical Model, in Phase Transitions and Critical Phenomena, vol. 2, C. Domb and M.S. Green eds., Academic Press (1972), p. 375.

[15] K.G. Wilson, The Renormalization Group: Critical Phenomena and the Kondo Problem, Rev. Mod. Phys. 47 (1975) 773 [inSPIRE].

[16] D.F. Litim and J.M. Pawlowski, On Gauge Invariant Wilsonian Flows, in The Exact Renormalization Group, A. Krasnitz, Y.A. Kubyshin, R. Potting and P. Sa. eds., World Scientific, Singapore (1999), p. 168 [hep-th/9901063] [INSPIRE]. 
[17] C. Bagnuls and C. Bervillier, Exact renormalization group equations. An Introductory review, Phys. Rept. 348 (2001) 91 [hep-th/0002034] [INSPIRE].

[18] J. Berges, N. Tetradis and C. Wetterich, Nonperturbative renormalization flow in quantum field theory and statistical physics, Phys. Rept. 363 (2002) 223 [hep-ph/0005122] [INSPIRE].

[19] J. Polonyi, Lectures on the functional renormalization group method, Central Eur. J. Phys. 1 (2004) 1 [hep-th/0110026] [INSPIRE].

[20] B. Delamotte, Introduction to the Non-Perturbative Renormalization Group, in Order, disorder and criticality. Advanced problems of phase transition theory, Y. Holovatch ed., World Scientific, Singapore (2007).

[21] B. Delamotte, An Introduction to the nonperturbative renormalization group, in Renormalization Group and Effective Field Theory Approaches to Many-Body Systems, Lect. Notes Phys. 852 (2012) 49 [cond-mat/0702365] [INSPIRE].

[22] H. Gies, Introduction to the functional $R G$ and applications to gauge theories, Lect. Notes Phys. 852 (2012) 287 [hep-ph/0611146] [INSPIRE].

[23] O.J. Rosten, Fundamentals of the Exact Renormalization Group, Phys. Rept. 511 (2012) 177 [arXiv: 1003.1366] [INSPIRE].

[24] H. Ballhausen, J. Berges and C. Wetterich, Critical phenomena in continuous dimension, Phys. Lett. B 582 (2004) 144 [hep-th/0310213] [INSPIRE].

[25] A. Codello, N. Defenu and G. D'Odorico, Critical exponents of $O(N)$ models in fractional dimensions, arXiv:1410.3308 [INSPIRE].

[26] S.-B. Liao, J. Polonyi and M. Strickland, Optimization of renormalization group flow, Nucl. Phys. B 567 (2000) 493 [hep-th/9905206] [INSPIRE].

[27] D.F. Litim, Critical exponents from optimized renormalization group flows, Nucl. Phys. B 631 (2002) 128 [hep-th/0203006] [INSPIRE].

[28] D.F. Litim, Universality and the renormalisation group, JHEP 07 (2005) 005 [hep-th/0503096] [INSPIRE].

[29] L. Canet, B. Delamotte, D. Mouhanna and J. Vidal, Optimization of the derivative expansion in the nonperturbative renormalization group, Phys. Rev. D 67 (2003) 065004 [hep-th/0211055] [INSPIRE].

[30] L. Canet, B. Delamotte, D. Mouhanna and J. Vidal, Nonperturbative renormalization group approach to the Ising model: A derivative expansion at order $\partial^{4}$, Phys. Rev. B 68 (2003) 064421 [hep-th/0302227] [INSPIRE].

[31] J.-P. Blaizot, R. Mendez Galain and N. Wschebor, A New method to solve the non perturbative renormalization group equations, Phys. Lett. B 632 (2006) 571 [hep-th/0503103] [INSPIRE].

[32] C. Bervillier, B. Boisseau and H. Giacomini, Analytical approximation schemes for solving exact renormalization group equations in the local potential approximation, Nucl. Phys. B 789 (2008) 525 [arXiv:0706.0990] [INSPIRE].

[33] C. Bervillier, B. Boisseau and H. Giacomini, Analytical approximation schemes for solving exact renormalization group equations. II. Conformal mappings, Nucl. Phys. B 801 (2008) 296 [arXiv: 0802.1970] [INSPIRE]. 
[34] F. Benitez, J.-P. Blaizot, H. Chaté, B. Delamotte, R. Méndez-Galain and N. Wschebor, Solutions of renormalization group flow equations with full momentum dependence, Phys. Rev. E 80 (2009) 030103 [arXiv:0901.0128] [InSPIRE].

[35] F. Benitez, J.-P. Blaizot, H. Chaté, B. Delamotte, R. Méndez-Galain and and N. Wschebor, Non-perturbative renormalization group preserving full-momentum dependence: implementation and quantitative evaluation, Phys. Rev. E 85 (2012) 026707 [arXiv:1110.2665] [INSPIRE].

[36] S. Nagy, Critical exponents of the $O(N)$ model in the infrared limit from functional renormalization, Phys. Rev. D 86 (2012) 085020 [arXiv:1201.1625] [INSPIRE].

[37] J.-M. Caillol, Critical line of the $\Phi^{4}$ theory on a simple cubic lattice in the local potential approximation, Nucl. Phys. B 865 (2012) 291 [arXiv:1207.4014] [INSPIRE].

[38] A.S. Kapoyannis and N. Tetradis, Quantum mechanical tunneling and the renormalization group, Phys. Lett. A 276 (2000) 225 [hep-th/0010180] [INSPIRE].

[39] D. Zappalà, Improving the renormalization group approach to the quantum mechanical double well potential, Phys. Lett. A 290 (2001) 35 [quant-ph/0108019] [INSPIRE].

[40] S. Nagy and K. Sailer, Functional renormalization group for quantized anharmonic oscillator, Annals Phys. 326 (2011) 1839 [arXiv: 1009.4041] [INSPIRE].

[41] N. Tetradis and C. Wetterich, Critical exponents from effective average action, Nucl. Phys. B 422 (1994) 541 [hep-ph/9308214] [INSPIRE].

[42] M. D'Attanasio and T.R. Morris, Large- $N$ and the renormalization group, Phys. Lett. B 409 (1997) 363 [hep-th/9704094] [INSPIRE].

[43] C. Wetterich, Average Action and the Renormalization Group Equations, Nucl. Phys. B $\mathbf{3 5 2}$ (1991) 529 [INSPIRE].

[44] C. Wetterich, Exact evolution equation for the effective potential, Phys. Lett. B 301 (1993) 90 [INSPIRE].

[45] T.R. Morris, The Exact renormalization group and approximate solutions, Int. J. Mod. Phys. A 9 (1994) 2411 [hep-ph/9308265] [INSPIRE].

[46] I. Nándori, Functional renormalization group with a compactly supported smooth regulator function, JHEP 04 (2013) 150 [arXiv:1208.5021] [INSPIRE].

[47] N. Tetradis and C. Wetterich, Scale dependence of the average potential around the maximum in $\varphi^{4}$ theories, Nucl. Phys. B 383 (1992) 197 [InSPIRE].

[48] S. Nagy, I. Nándori, J. Polonyi and K. Sailer, Functional renormalization group approach to the Sine-Gordon model, Phys. Rev. Lett. 102 (2009) 241603 [arXiv:0904.3689] [InSPIRE].

[49] J. Braun, H. Gies and D.D. Scherer, Asymptotic safety: a simple example, Phys. Rev. D 83 (2011) 085012 [arXiv: 1011.1456] [INSPIRE].

[50] S. Nagy, Degeneracy induced scaling of the correlation length for periodic models, Nucl. Phys. B 864 (2012) 226 [arXiv:1204.0440] [INSPIRE].

[51] S. Nagy, Lectures on renormalization and asymptotic safety, Annals Phys. 350 (2014) 310 [arXiv: 1211.4151] [INSPIRE].

[52] S. Nagy and K. Sailer, Interplay of fixed points in scalar models, Int. J. Mod. Phys. A 28 (2013) 1350130 [arXiv:1012.3007] [INSPIRE]. 
[53] A. Codello, G. D'Odorico and C. Pagani, A functional RG equation for the c-function, JHEP 07 (2014) 040 [arXiv: 1312 .7097] [INSPIRE].

[54] J. Zinn-Justin, Quantum Field Theory and Critical Phenomena, Clarendon Press, Oxford (1989).

[55] E. Marchais, Infrared properties of scalar field theories, Ph.D. Thesis, University of Sussex, Brighton, U.K. and online at http://sro.sussex.ac.uk/45244/.

[56] T.R. Morris, On truncations of the exact renormalization group, Phys. Lett. B 334 (1994) 355 [hep-th/9405190] [INSPIRE].

[57] A. Codello, Scaling Solutions in Continuous Dimension, J. Phys. A 45 (2012) 465006 [arXiv: 1204.3877] [INSPIRE].

[58] T.R. Morris, Properties of derivative expansion approximations to the renormalization group, Int. J. Mod. Phys. B 12 (1998) 1343 [hep-th/9610012] [InSPIRE].

[59] V.L. Berezinskii, Destruction of long-range order in one-dimensional and two-dimensional system possessing a continuous symmetry group, II. Quantum systems, Zh. Eksp. Teor. Fiz. 61 (1971) 1144 [Sov. Phys. JETP 34 (1972) 610].

[60] J.M. Kosterlitz and D.J. Thouless, Ordering, metastability and phase transitions in two-dimensional systems, J. Phys. C 6 (1973) 1181 [INSPIRE].

[61] M. Gräter and C. Wetterich, Kosterlitz-Thouless phase transition in the two-dimensional linear $\sigma$-model, Phys. Rev. Lett. 75 (1995) 378 [hep-ph/9409459] [INSPIRE].

[62] I. Nándori, J. Polonyi and K. Sailer, On the renormalization of periodic potentials, Phys. Rev. D 63 (2001) 045022 [hep-th/9910167] [INSPIRE].

[63] G. von Gersdorff and C. Wetterich, Nonperturbative renormalization flow and essential scaling for the Kosterlitz-Thouless transition, Phys. Rev. B 64 (2001) 054513 [hep-th/0008114] [INSPIRE].

[64] I. Nándori, I.G. Márián and V. Bacsó, Spontaneous symmetry breaking and optimization of functional renormalization group, Phys. Rev. D 89 (2014) 047701 [arXiv:1303.4508] [INSPIRE].

[65] I.G. Márián, U.D. Jentschura and I. Nándori, The numerically optimized regulator and the functional renormalization group, J. Phys. G 41 (2014) 055001 [arXiv:1311.7377] [INSPIRE].

[66] D.F. Litim, Optimization of the exact renormalization group, Phys. Lett. B 486 (2000) 92 [hep-th/0005245] [INSPIRE].

[67] D.F. Litim, Optimized renormalization group flows, Phys. Rev. D 64 (2001) 105007 [hep-th/0103195] [INSPIRE].

[68] D.F. Litim, Derivative expansion and renormalization group flows, JHEP 11 (2001) 059 [hep-th/0111159] [INSPIRE]. 